\title{
Neuroimaging studies on cognitive impairment due to cerebral small vessel disease
}

To cite: Du J, Xu Q. Neuroimaging studies on cognitive impairment due to cerebral small vessel disease. Stroke and Vascular Neurology 2019;4: e000209. doi:10.1136/ svn-2018-000209

Received 5 November 2018 Revised 23 February 2019 Accepted 18 March 2019 Published Online First 5 April 2019

\section{Check for updates}

(C) Author(s) (or their employer(s)) 2019. Re-use permitted under CC BY-NC. No commercial re-use. See rights and permissions. Published by BMJ.

${ }^{1}$ Neurology, Shanghai Jiao Tong University School of Medicine Affiliated Renji Hospital,

Shanghai, China

${ }^{2}$ Health Manage Center, Shanghai Jiao Tong University School of Medicine Affiliated Renji Hospital, Shanghai, China

Correspondence to

Dr Qun Xu; xuqun628@163.com

Jing Du, ${ }^{1}$ Qun $X u^{\oplus 2}$

\section{ABSTRACT}

Vascular cognitive impairment (VCI) is a major contributor to age-related dementing illnesses which imposes a tremendous burden on families and society. It is a heterogeneous group of brain disorders. However, cerebral small vessel disease (CSVD) accounts for about 50\%-70\% of $\mathrm{VCl}$, which represented a more homogeneous subtype of $\mathrm{VCl}$. Advanced multimodal neuroimaging techniques like brain network connectome analyses are currently applied to explore the underlying mechanism of VCI. Some progress in the field of structural and functional brain network researches on a poststroke longitudinal CSVD cohort (Renji CSVD Cohort Study) was reported. Global and regional brain network characters were compared between patients with CSVD and healthy control. It suggested that distributed brain structural network disruption may play a pivot role in the cognitive decline. The results showed that brain structural network characters have distinctive differentiating capacity on the cognition of patients with CSVD.

\section{CEREBRAL SMALL VESSEL DISEASE AND VASCULAR COGNITIVE IMPAIRMENT}

The term cerebral small vessel disease (CSVD) encompasses all the pathological processes that affect the small vessels of the brain, including small arteries and arterioles, and capillaries and small veins. ${ }^{12}$ Arteriolosclerosis (age-related or vascular risk-factor-related small vessel diseases) is sporadic and the most prevalent form of CSVD. It is characterised pathologically by lipohyalinosis, microaneurysm, microatheroma and fibrinoid necrosis. ${ }^{1}$ CSVD may present with the insidious onset of minor stroke(s), but it will gradually lead to the outcomes such as cognitive impairment, physical disabilities and emotion change in a long time with various stages and a progressive course. It accounts for 10\%-30\% of stroke cases and $50 \%-70 \%$ of vascular cognitive impairment (VCI). ${ }^{3}{ }^{4}$ CSVD has been proved as a slowly progressing disease that destroyed frontal-subcortical networks, leading to various frontal symptoms, especially the decreased executive and memory function with relatively reserved recognition. ${ }^{5}$ Hence, CSVD produces the most prevalent subtype of VCI, the subcortical ones.

\section{NEUROIMAGING STUDIES ON VCI DUE TO CSVD}

There are few animal models which have been developed to study the form of VCI due to CSVD. ${ }^{6}$ Furthermore, small vessels cannot be visualised in vivo and the pathological evidence of CSVD is also limited. Therefore, neuroimaging is adopted as an important method for the diagnosis and research of CSVD. ${ }^{78}$ With the rapid development of novel neuroimaging techniques, much progress has been made. Currently, the Standards for Reporting Vascular changes on Neuroimaging criteria is widely applied to evaluate structural features seen on MRI, including recent small subcortical infarcts, lacunes, white matter hyperintensities, enlarged perivascular spaces, microbleeds and brain atrophy. ${ }^{9}$ These individual MRI markers of CSVD are inter-related, and their respective correlations with cognition have been demonstrated, ${ }^{10-13}$ while some of the results are reported inconsistently. ${ }^{7} 14$ Therefore, it exists the unmet need to better quantify total CSVD burden and relate it to cognition and dementia.

As the conventional focal lesions usually affect remote structural and functional network connections, CSVD is now considered as a global rather than a focal disease. ${ }^{15}$ SVD score is a visual semiquantitative approach which combines such lesion burdens in a semiquantitative manner as proposed by Staals $e t$ $a l^{1617}$ However, the relationship of SVD score and cognitive function has not been widely examined in cohorts from different clinical settings. $^{18}$

Considering that the semiquantitative SVD score does not identify the extent and location of different lesions, whether this composite index really indicates an underlying latent disease construct or not is untested. It is meaningful but challenging to find a composite convincing method to quantify CSVD burden with neuroimaging techniques.

Brain network is usually defined as a large-scale complex network mapping the brain structure and function. It has been proved to be economical and efficient with 
the 'small world' characteristics. ${ }^{19}$ It is an intermediate state of a complex network, which achieves a balance between functional integration and functional differentiation. Brain network connectome analyses brain lesions from both integrated and separated perspective by the method of graph theory. Several studies have demonstrated that brain network characters have advantages of explaining cognitive dysfunction than conventional structural lesions. ${ }^{20}{ }^{21}$ It may become a reliable surrogate biomarker for CSVD. ${ }^{22}$ The results of a 5-year prospective hospital-based cohort study demonstrated that network disruption played a pivotal role in the genesis of dementia in CSVD, suggesting that brain network measures have potentials of surrogate markers of subcortical VCI. ${ }^{21}$ Lawrence $e t a l^{22}$ constructed a cross-sectional study comparing the brain network measures between patients with CSVD and healthy individuals. The results indicated that network connectivity was significantly reduced in patients with CSVD and such disruption was associated with disease severity and cognitive function. Some research applying the mediation analysis were conducted to explore the relationship among structural lesions, brain networks and cognitive function. ${ }^{142324}$ The results of these research provided some evidence of the underlying mechanism in CSVD showing that the associations between structural lesions and cognition was fully or partly mediated by the destruction of brain networks.

\section{NEUROIMAGING STUDY ON RENJI CSVD COHORT}

Renji CSVD Cohort Study (RCCS; http://www.clinicaltrials.gov, NCT 03544801) is a longitudinal cohort study, which recruits patients with CSVD consecutively from the stroke clinic at the Department of Neurology, Renji Hospital, an affiliated teaching institution of School of Medicine, Shanghai JiaoTong University since 2015. One of the aims of this study is to investigate the mechanism of the occurrence and development of VCI due to CSVD by multimodal MRI. It was found that the correlation existed between white matter hyperintensity and cognitive dysfunction. ${ }^{25}{ }^{26}$ Furthermore, arteriosclerotic microbleeds and periventricular white matter hyperintensity seem to be independent MRI surrogated markers in the early stage of cognitive impairment in CSVD ${ }^{27}$ Abnormal reductions in cortical cerebral blood flow (CBF) have been identified in VCI due to CSVD. However, little is known about the pattern of CBF reduction in relation to the degree of cognitive impairment. A study on $\mathrm{CBF}$ alterations assessed by three-dimensional arterial spin labelling was performed in RCCS patients to detect the relationship of regional CBF with cognition in CSVD. The results suggested that cerebral perfusion deficit in the temporal and frontal lobe, hippocampus, thalamus and insula was correlated with the degree of cognitive impairment. ${ }^{28}$ Recently, functional with structural brain network analysis was conducted on our cohort. A resting-state functional MRI study demonstrated that functional connectivity alteration was observed in patients with CSVD. Resting-state functional connectivity between the left thalamus and the posterior cingulate cortex indicates the severity of cognitive impairment. The frontal lobe and other subcortical brain sites play an important role in the pathogenesis of VCI due to CSVD..$^{29}$ Evidence suggested that distributed structural brain network disruption may play a pivotal role in the cognitive decline. The inter-relationships between cerebral vascular brain injuries (CVBIs), structural network disruptions and cognitive dysfunction were explored using mediation analysis recently on RCCS. It was found that structural network disruptions play an intermediate role between the CVBIs and the cognitive decline. In another study of RCCS, several global and nodal brain network characters were compared between patients with CSVD and healthy controls. The preliminary results showed that brain structural network characters differentiate CSVD from healthy controls and distinguish patients with CSVD with different cognitive status.

\section{CONCLUSIONS AND FUTURE PERSPECTIVES}

VCI is a major contributor to age-related dementing illnesses. CSVD is the leading cause of VCI which presents with predominant disruption of attention and executive function. It is particularly important for early detection and prevention since no specific treatment has been found to be effective until now. Multimodal neuroimaging techniques are currently important investigation tools in clinical studies. Brain network characters are now emerging as sensitive markers in cognitive deterioration in CSVD. Advanced structural with functional brain network analysis on longitudinal cohorts with different clinical settings are needed to explore the mechanism of VCI in CSVD in the future.

Contributors This paper was drafted by JD and was then amended and approved by $Q X$.

Funding This study was supported by grants from the National Key Research and Development Program of China (2016YFC1300600), The Innovation Action Project of Shanghai Committee of Science and Technology (17JC404100), The Project of Collaborative Innovation Center of Translational Medicine (TM201808) and SJTUUNSW Collaborative Research Funding.

Competing interests None declared.

Patient consent for publication Not required.

Provenance and peer review Not commissioned; externally peer reviewed.

Open access This is an open access article distributed in accordance with the Creative Commons Attribution Non Commercial (CC BY-NC 4.0) license, which permits others to distribute, remix, adapt, build upon this work non-commercially, and license their derivative works on different terms, provided the original work is properly cited, appropriate credit is given, any changes made indicated, and the use is non-commercial. See: http://creativecommons.org/licenses/by-nc/4.0/.

\section{REFERENCES}

1. Pantoni L. Cerebral small vessel disease: from pathogenesis and clinical characteristics to therapeutic challenges. Lancet Neurol 2010;9:689-701.

2. Østergaard L, Engedal TS, Moreton F, et al. Cerebral small vessel disease: capillary pathways to stroke and cognitive decline. J Cereb Blood Flow Metab 2016;36:302-25. 
3. Shi Y, Wardlaw JM. Update on cerebral small vessel disease: a dynamic whole-brain disease. Stroke Vasc Neurol 2016;1:83-92.

4. Zhang AJ, Yu XJ, Wang M. The clinical manifestations and pathophysiology of cerebral small vessel disease. Neurosci Bull 2010;26:257-64.

5. Jellinger KA. Pathology and pathogenesis of vascular cognitive impairment-a critical update. Front Aging Neurosci 2013;5:17.

6. Yang Y, Kimura-Ohba S, Thompson J, et al. Rodent Models of Vascular Cognitive Impairment. Trans/ Stroke Res 2016;7:407-14.

7. Banerjee G, Wilson D, Jäger HR, et al. Novel imaging techniques in cerebral small vessel diseases and vascular cognitive impairment. Biochim Biophys Acta 2016;1862:926-38.

8. Chen X, Wang J, Shan Y, et al. Cerebral small vessel disease: neuroimaging markers and clinical implication. J Neurol 2018 [Epub ahead of print 5 Oct 2018].

9. Wardlaw JM, Smith EE, Biessels GJ, et al. Neuroimaging standards for research into small vessel disease and its contribution to ageing and neurodegeneration. Lancet Neurol 2013;12:822-38.

10. Tuladhar AM, van Norden AG, de Laat KF, et al. White matter integrity in small vessel disease is related to cognition. Neuroimage Clin 2015;7:518-24.

11. Makin SD, Turpin S, Dennis MS, et al. Cognitive impairment after lacunar stroke: systematic review and meta-analysis of incidence, prevalence and comparison with other stroke subtypes. $J$ Neurol Neurosurg Psychiatry 2013;84:893-900.

12. Patel B, Lawrence AJ, Chung AW, et al. Cerebral microbleeds and cognition in patients with symptomatic small vessel disease. Stroke 2013;44:356-61.

13. Arba F, Quinn TJ, Hankey GJ, et al. Enlarged perivascular spaces and cognitive impairment after stroke and transient ischemic attack. Int $J$ Stroke 2018;13:47-56.

14. Banerjee G, Jang H, Kim HJ, et al. Total MRI small vessel disease burden correlates with cognitive performance, cortical atrophy, and network measures in a memory clinic population. J Alzheimers Dis 2018;63:1485-97.

15. Ter Telgte A, van Leijsen EMC, Wiegertjes $\mathrm{K}$, et al. Cerebral small vessel disease: from a focal to a global perspective. Nat Rev Neurol 2018;14:387-98.

16. Huijts $M$, Duits $A$, van Oostenbrugge RJ, et al. Accumulation of MRI Markers of Cerebral Small Vessel Disease is Associated with Decreased Cognitive Function. A Study in First-Ever Lacunar Stroke and Hypertensive Patients. Front Aging Neurosci 2013;5:72.
17. Staals J, Makin SD, Doubal FN, et al. Stroke subtype, vascular risk factors, and total MRI brain small-vessel disease burden. Neurology 2014;83:1228-34.

18. Staals J, Booth T, Morris Z, et al. Total MRI load of cerebral small vessel disease and cognitive ability in older people. Neurobiol Aging 2015;36:2806-11.

19. He Y, Chen ZJ, Evans AC. Small-world anatomical networks in the human brain revealed by cortical thickness from MRI. Cereb Cortex 2007;17:2407-19.

20. Tuladhar AM, van Dijk E, Zwiers MP, et al. Structural network connectivity and cognition in cerebral small vessel disease. Hum Brain Mapp 2016;37:300-10.

21. Tuladhar AM, van Uden IW, Rutten-Jacobs LC, et al. Structural network efficiency predicts conversion to dementia. Neurology 2016;86:1112-9.

22. Lawrence AJ, Chung AW, Morris RG, et al. Structural network efficiency is associated with cognitive impairment in small-vessel disease. Neurology 2014;83:304-11.

23. Du J, Wang Y, Zhi N, et al. Structural brain network measures are superior to vascular burden scores in predicting early cognitive impairment in post stroke patients with small vessel disease. Neuroimage Clin 2019;22:101712.

24. Heinen $\mathrm{R}$, Vlegels $\mathrm{N}$, de Bresser $\mathrm{J}$, et al. The cumulative effect of small vessel disease lesions is reflected in structural brain networks of memory clinic patients. Neuroimage Clin 2018;19.

25. Wang Y, Cao W, Sun Y, et al. White matter integrity in subcortical vascular cognitive impairment: A multimodal structural MRI Study. Curr Alzheimer Res 2017;14:991-9.

26. Xu Q, Zhou Y, Li YS, et al. Diffusion tensor imaging changes correlate with cognition better than conventional MRI findings in patients with subcortical ischemic vascular disease. Dement Geriatr Cogn Disord 2010;30:317-26.

27. Cao WW, Wang Y, Dong Q, et al. Deep microbleeds and periventricular white matter disintegrity are independent predictors of attention/executive dysfunction in non-dementia patients with small vessel disease. Int Psychogeriatr 2017;29:793-803.

28. Sun Y, Cao W, Ding W, et al. Cerebral blood flow alterations as assessed by 3D ASL in cognitive impairment in patients with subcortical vascular cognitive impairment: a marker for disease severity. Front Aging Neurosci 2016;8:211.

29. Ding W, Cao W, Wang Y, et al. Altered functional connectivity in patients with subcortical vascular cognitive impairment--a restingstate functional magnetic resonance imaging study. PLoS One 2015;10:e0138180. 